\title{
Dependence of effective spectrum width of synchrotron radiation on particle energy
}

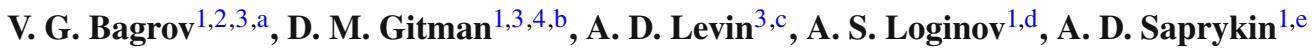 \\ ${ }^{1}$ Department of Physics, Tomsk State University, Tomsk, Russia \\ ${ }^{2}$ Institute of High Current Electronics, SB RAS, Tomsk, Russia \\ ${ }^{3}$ Institute of Physics, University of Sao Paulo, Sao Paulo, Brazil \\ ${ }^{4}$ P.N. Lebedev Physical Institute, Moscow, Russia
}

Received: 7 April 2017 / Accepted: 28 April 2017 / Published online: 24 May 2017

(C) The Author(s) 2017. This article is an open access publication

\begin{abstract}
In the classical theory of synchrotron radiation, for the exact quantitative characterization of spectral properties, the concept of effective spectral width is introduced. In the first part of our work, published in EJPC 75 (2015), the effective spectral width as a function of the energy $E$ of the radiating particle was obtained only in the ultra-relativistic approximation. In this article, which can be considered as a natural continuation of this work, a complete investigation is presented of the dependence of the effective width of the synchrotron radiation spectrum on energy for any values of $E$ and for all the polarization components of the radiation. Numerical calculations were carried out for an effective width not exceeding 100 harmonics.
\end{abstract}

\section{Introduction}

As one of the major quantitative characteristics of spectral distributions for electromagnetic radiation, one commonly uses the concept of spectral half-width. For spectral distributions having a sharp maximum, the spectral half-width is the most informative physical characteristic.

However, once a spectral distribution has no pronounced maximum, the spectral half-width ceases to be an adequate quantitative characteristic. In particular, this is exactly the case of spectral distributions for synchrotron radiation (SR), and therefore the SR spectral half-width has neither been calculated theoretically, nor measured experimentally.

\footnotetext{
a e-mail: bagrov@phys.tsu.ru

b e-mail: gitman@if.usp.br

c e-mail: alevin@if.usp.br

d e-mail: loginov@phys.tsu.ru

e e-mail: alexx.saprykin.phys@gmail.com
}

Instead of the spectral half-width, the present study proposes the introduction of a new precise quantitative characteristic of SR spectral distributions: the effective spectral width. We show how this quantity can be calculated theoretically, and which physically relevant information can be obtained using this quantity.

At present, the theory of synchrotron radiation is a fairly well-developed area of theoretical physics. Its main elements are described in monographs (e.g. [1-7]) and numerous articles. The high polarization degree of radiation and unique structure of spectral distributions are among the most important SR physical features. All theoretically predictable SR properties have been confirmed by experiment. The development of SR theory allows one not only to predict radiation characteristics qualitatively, but also to offer exact quantitative characteristics of physically important properties. For example, the high degree of SR polarization was qualitatively predicted by theory more than half a century ago (see, e.g., [1]); precise quantitative characteristics for linear polarization were also obtained, whereas such quantitative characteristics for circular polarization were obtained much later [8-11].

In order to set up the problem, we now present some wellknown expressions of the classical SR theory for the physical characteristics of synchrotron radiation, which can be found in [1-7].

The spectral-angular distribution for radiation power of SR polarization components can be written as

$$
W_{s}=W \sum_{\nu=1}^{\infty} \int_{0}^{\pi} f_{s}(\beta ; \nu, \theta) \sin \theta \mathrm{d} \theta .
$$

Here, the following notation is used: $\theta$ is the angle between the control magnetic field strength and the radiation field pulse; $v$ is the number of an emitted harmonic; the charge 
orbital motion rate is $v=c \beta$, where $c$ is the speed of light; $W$ is the total radiated power of unpolarized radiation, which can be written as

$W=\frac{2}{3} \frac{c e^{2}}{R^{2}}\left(\gamma^{2}-1\right)^{2}=\frac{2 e^{4} H^{2}\left(\gamma^{2}-1\right)}{3 m_{0}^{2} c^{3}}, \quad \gamma=\frac{1}{\sqrt{1-\beta^{2}}}$,

where $e$ is the particle charge; $R$ is the orbit radius; $H$ is the control field strength; $m_{0}$ is the charge rest mass; $\gamma$ is the relativistic factor. The index $s$ numbers the polarization components: $s=2$ corresponds to the $\sigma$-component of linear polarization; $s=3$ corresponds to the $\pi$-component of linear polarization; $s=1$ corresponds to right-hand circular polarization; $s=-1$ corresponds to left-hand circular polarization; $s=0$ corresponds to the power of unpolarized radiation. The functions $f_{s}(\beta ; v, \theta)$ have the form [1-7]

$f_{2}(\beta ; v, \theta)=\frac{3 v^{2}}{2 \gamma^{4}} J_{v}^{\prime 2}(x) ; \quad f_{3}(\beta ; v, \theta)$

$$
=\frac{3 v^{2}}{2 \gamma^{4}} \frac{\cos ^{2} \theta}{\beta^{2} \sin ^{2} \theta} J_{\nu}^{2}(x)
$$

$f_{ \pm 1}(\beta ; v, \theta)=\frac{3 v^{2}}{4 \gamma^{4}}\left[J_{v}^{\prime}(x) \pm \varepsilon \frac{\cos \theta}{\beta \sin \theta} J_{v}(x)\right]^{2} ;$

$$
x=\nu \beta \sin \theta ; \quad \varepsilon=-\frac{e}{|e|} ;
$$

$f_{0}(\beta ; v, \theta)=f_{2}(\beta ; v, \theta)+f_{3}(\beta ; v, \theta)=f_{1}(\beta ; \nu, \theta)$

$$
+f_{-1}(\beta ; v, \theta) \text {. }
$$

Here, $J_{v}(x)$ and $J_{v}^{\prime}(x)$ are the Bessel functions and their derivatives. In what follows, the case of an electron is considered, which corresponds to $\varepsilon=1$.

\section{Spectral distribution for polarization components of synchrotron radiation in the upper half-space}

It is well known [1-7] that the angle range $0 \leqslant \theta<\pi / 2$ (this range will be called the upper half-space) is dominated by right-hand circular polarization, and the angle range $\pi / 2<\theta \leqslant \pi$ (this range will be called the lower half-space) is dominated by left-hand circular polarization (exact quantitative characteristics of SR properties were first obtained in $[8-11])$. However, if we integrate in (1) over $\theta(0 \leqslant \theta \leqslant \pi)$, then the differences in the spectral distribution of right- and left-hand circular polarizations disappear. To reveal these differences, the expressions of Eq. (1) can be represented by

$$
\begin{aligned}
W_{s} & =W\left[\Phi_{s}^{(+)}(\beta)+\Phi_{s}^{(-)}(\beta)\right] ; \Phi_{s}^{(+)}(\beta) \\
& =\sum_{\nu=1}^{\infty} F_{s}^{(+)}(\beta ; \nu), \Phi_{s}^{(-)}(\beta)=\sum_{\nu=1}^{\infty} F_{s}^{(-)}(\beta ; \nu) ;
\end{aligned}
$$

$$
\begin{aligned}
& F_{s}^{(+)}(\beta ; v)=\int_{0}^{\pi / 2} f_{s}(\beta ; v, \theta) \sin \theta \mathrm{d} \theta \\
& F_{s}^{(-)}(\beta ; v)=\int_{\pi / 2}^{\pi} f_{S}(\beta ; v, \theta) \sin \theta \mathrm{d} \theta
\end{aligned}
$$

and it suffices to study the properties of the functions $F_{s}^{(+)}(\beta ; v)$ (respectively, the properties of functions $\Phi_{s}^{(+)}$ $(\beta)$ ), due to the evident relations

$$
\begin{aligned}
& F_{s}^{(-)}(\beta ; v)=F_{s}^{(+)}(\beta ; v), \quad \Phi_{s}^{(-)}(\beta)=\Phi_{s}^{(+)}(\beta), \\
& \quad s=0,2,3 ; \\
& F_{1}^{(-)}(\beta ; v)=F_{-1}^{(+)}(\beta ; v), \quad \Phi_{1}^{(-)}(\beta)=\Phi_{-1}^{(+)}(\beta), \\
& F_{-1}^{(-)}(\beta ; v)=F_{1}^{(+)}(\beta ; v), \quad \Phi_{-1}^{(-)}(\beta)=\Phi_{1}^{(+)}(\beta) .
\end{aligned}
$$

Integration over $\theta$ in the upper half-space $0 \leqslant \theta \leqslant \pi / 2$ in (4) can be carried out exactly, yielding the expressions

$$
\begin{aligned}
& F_{2}^{(+)}(\beta ; v) \\
& =\frac{3 v}{4 \gamma^{4} \beta^{3}}\left[2 \beta^{2} J_{2 v}^{\prime 2} \int_{0}^{2 v \beta} J_{2 v}(x) \mathrm{d} x-2 v \beta \int_{0}^{2 v \beta} \frac{J_{2 v}(x)}{x} \mathrm{~d} x\right] \\
& F_{3}^{(+)}(\beta ; v) \\
& =\frac{3 v}{4 \gamma^{4} \beta^{3}}\left[2 v \beta \int_{0}^{2 v \beta} \frac{J_{2 v}(x)}{x} \mathrm{~d} x-\int_{0}^{2 v \beta} J_{2 v}(x) \mathrm{d} x\right] \\
& F_{0}^{(+)}(\beta ; v)=F_{2}^{(+)}(\beta ; v)+F_{3}^{(+)}(\beta ; v) \\
& \left.=\frac{3 v}{4 \gamma^{4} \beta^{3}}\left[2 \beta^{2} J_{2 v}^{\prime 2}\right) \int_{0}^{2 v \beta} J_{2 v}(x) \mathrm{d} x\right] \\
& F_{ \pm 1}^{(+)}(\beta ; v)=\frac{1}{2} F_{0}^{(+)}(\beta ; v) \pm \frac{3 v J_{v}^{2}(v \beta)}{4 \gamma^{4} \beta^{2}} .
\end{aligned}
$$

The sums over the harmonics $v$ in (4) can also be calculated exactly, yielding the expressions

$$
\begin{aligned}
& \Phi_{2}^{(+)}(\beta)=\frac{6+\beta^{2}}{16}, \Phi_{3}^{(+)}(\beta)=\frac{2-\beta^{2}}{16}, \Phi_{0}^{(+)}(\beta)=\frac{1}{2}, \\
& \Phi_{ \pm 1}^{(+)}(\beta)=\frac{1}{4}\left[1 \pm \frac{3}{4} \chi_{1}(\beta)\right] .
\end{aligned}
$$

The function $\chi_{1}(\beta)$ introduced above was defined and studied in [8]. In particular, it was shown [8] that in the segment $0 \leqslant \beta \leqslant 1$ the function $\chi_{1}(\beta)$ is finite and decreases monotonously; at the end of this segment, it takes the following values:

$\chi_{1}(0)=1, \quad \chi_{1}(1)=\frac{4}{\pi \sqrt{3}}$. 


\section{Effective spectral width for polarization components of synchrotron radiation}

As one of the quantitative characteristics of physical properties for spectral distributions of SR polarization components, we propose to introduce the concept of effective spectral width $\Lambda_{s}(\beta)$. Let us define $\Lambda_{s}(\beta)$ as follows.

For each fixed value of $\beta$, we examine the quantities

$$
\begin{aligned}
\widetilde{\Phi}_{s} & \left(\beta ; v^{(1)}, v^{(2)}\right) \\
\quad= & \sum_{\nu=v^{(1)}}^{v^{(2)}} F_{s}^{(+)}(\beta ; v), \quad 1 \leqslant v^{(1)} \leqslant v^{(2)} \leqslant \infty .
\end{aligned}
$$

The following relations are obvious:

$$
\begin{gathered}
\Phi_{s}^{(+)}(\beta)=\widetilde{\Phi}_{s}\left(\beta ; v^{(1)}=1, v^{(2)}=\infty\right) ; \\
\Phi_{s}^{(+)}(\beta)>\widetilde{\Phi}_{s}\left(\beta ; v^{(1)} \geqslant 1, v^{(2)}<\infty\right) .
\end{gathered}
$$

Consider the set of values $v^{(1)}, v^{(2)}\left(1 \leqslant v^{(1)} \leqslant v^{(2)}<\infty\right)$ so as to satisfy the inequality

$\widetilde{\Phi}_{s}\left(\beta ; v^{(1)}, v^{(2)}\right) \geqslant \frac{1}{2} \Phi_{s}^{(+)}(\beta)$.

Obviously, such values $v^{(1)}, v^{(2)}$ do exist for any $\beta$ (for example, $v^{(1)}=1$ necessarily yields such a finite value for $\left.v^{(2)}\right)$. It is equally obvious that condition (11) alone is generally insufficient to determine the pair of values $v^{(1)}, v^{(2)}$. Let us now choose such $v_{s}^{(1)}(\beta), v_{s}^{(2)}(\beta)$ that the condition (11) should provide the minimum of the difference $v_{s}^{(2)}(\beta)-v_{s}^{(1)}(\beta)$, as well as the minimum of the non-negative value

$\widetilde{\Phi}_{s}\left(\beta ; v_{s}^{(1)}(\beta), v_{s}^{(2)}(\beta)\right)-\frac{1}{2} \Phi_{s}^{(+)}(\beta) \geqslant 0$.

The effective spectral width $\Lambda_{s}(\beta)$ is defined by the expression

$\Lambda_{s}(\beta)=v_{s}^{(2)}(\beta)-v_{s}^{(1)}(\beta)+1, \quad \Lambda_{s}(\beta) \geqslant 1$.

Consequently, the effective spectral width is the minimum spectral range that accounts for at least half of all the radiated power of a given polarization component. The harmonics $v_{s}^{(1)}(\beta)$ and $v_{s}^{(2)}(\beta)$ determine the beginning and the end of this minimum spectral range.

A definition equivalent to the one presented above for the effective spectral width can be given using the concept of partial contributions $P_{S}(\beta ; \nu)$ for individual spectral harmonics, introduced in [12]. Namely, we suppose, according to [12],

$P_{S}(\beta ; \nu)=\frac{F_{s}^{(+)}(\beta ; v)}{\Phi_{s}^{(+)}(\beta)}$.

Then (4) implies the property

$\sum_{\nu=1}^{\infty} P_{s}(\beta ; v)=1$.
We choose some values $v_{s}^{(1)}(\beta)$ and $v_{s}^{(2)}(\beta)$ such that the minimum difference $v_{s}^{(2)}(\beta)-v_{s}^{(1)}(\beta)$ should provide the minimum of the non-negative value

$$
\sum_{\nu=\nu_{s}^{(1)}(\beta)}^{\nu_{s}^{(2)}(\beta)} P_{s}(\beta ; \nu)-\frac{1}{2} \geqslant 0 .
$$

Introducing $\Lambda_{s}(\beta)$ in accordance with (13), we arrive at the following equivalent definition: effective spectral width is the minimum spectral range at which the sum of partial contributions for individual harmonics is not less than $1 / 2$.

In practice, the most interesting case is the ultra-relativistic limit $(\beta \approx 1$, equivalent to $\gamma \gg 1)$. In this case, the analytical study of effective spectral width and other physically interesting quantitative characteristics for spectral distributions of SR polarization components can be significantly extended. This study was carried out in [13].

Given a particular value of $\beta$ (or $\gamma$ ), it is a purely computational task to obtain the exact values of $\Lambda_{s}(\beta)$ and $v_{s}^{(1)}(\beta)$. In this article, we present a numerical study of the region $1 \leqslant \Lambda_{s}(\beta) \leqslant 100$. It is essential to observe the following. The effective width $\Lambda_{s}(\beta)$ is a positive integer, so there exists a range of $\beta$ (corresponding to a range of $\gamma$; hereinafter, we only indicate $\gamma)$ in which $\Lambda_{s}(\beta)$ is constant.

\section{Analysis of numerical results for effective spectral width of synchrotron radiation}

The main results of a numerical study for effective spectral width of SR polarization components are given by Table 1 . Calculation results for larger $\Lambda$ can be found in [14].

The numerical study is carried out as follows. For each type of polarization $s$, we examine the sequences of integers $\Lambda_{s}=1,2,3 \ldots$ and $v_{s}^{(1)}=1,2,3 \ldots$ (it is evident that $\left.v_{s}^{(2)}=v_{s}^{(1)}+\Lambda_{s}-1\right)$ and determine the regions of values $\gamma_{s}$ for which the condition (16) (equivalent to (12)) is satisfied. It is clear that the boundary points of possible regions for $\gamma_{s}$ can be found, according to (16), as solutions of the equations

$\sum_{l=0}^{\Lambda_{s}-1} P_{S}\left(\beta ; v_{s}^{(1)}+l\right)-\frac{1}{2}=0$.

The roots $\beta_{s}=\beta_{s}\left(\Lambda_{s}, v_{s}^{(1)}\right)$ of these equations determine the boundary points $\gamma_{s}=\gamma_{s}\left(\Lambda_{s}, v_{s}^{(1)}\right)$, in accordance with (2).

Let us consider in more detail the calculation method and the results pertaining to the $\sigma$ - component of linear polarization. These results are given in the column for $s=2$ of our Table. We denote $v_{2}^{(1)}=k=1,2,3 \ldots$. 


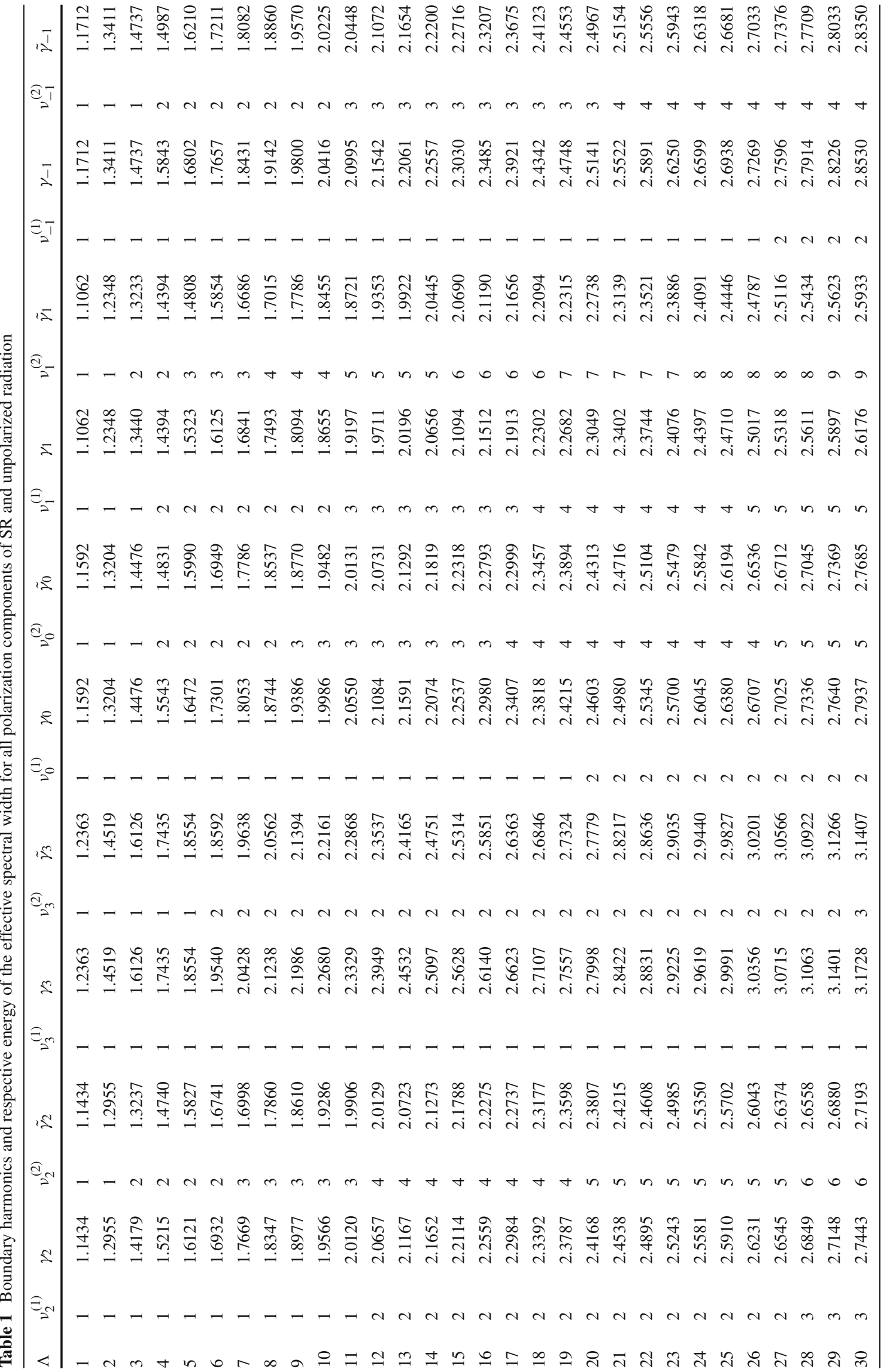




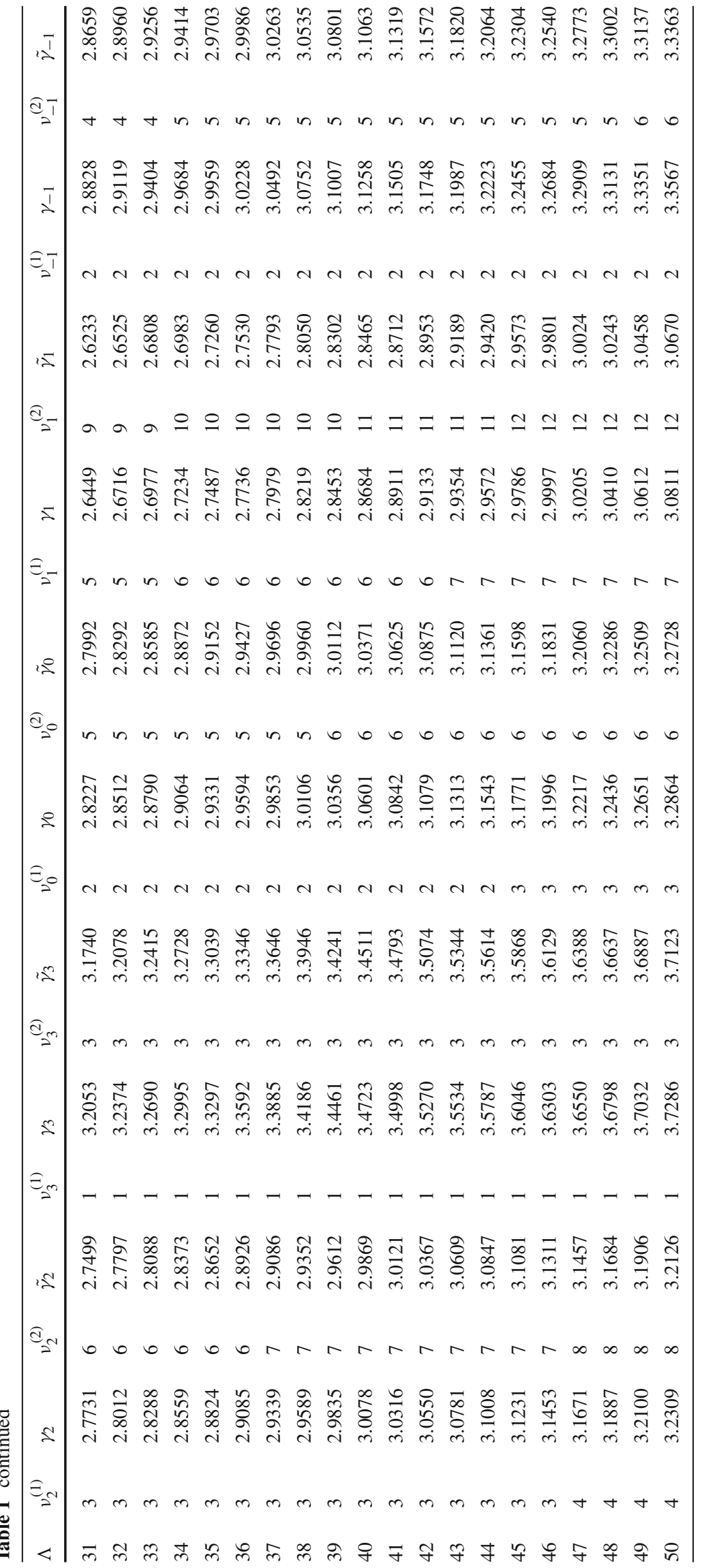


At the first step, we examine the smallest possible value $\Lambda_{2}=1$. In this case, Eq. (17) has the form

$P_{2}(\beta ; k)-\frac{1}{2}=0$

According to the results of [12], all partial contributions at $k \geqslant 2$ are such that $P_{2}(\beta ; k)<1 / 2$ for all the values of $\gamma$, whereas in the first harmonic $(k=1)$ such a region of values $\gamma$ does exist, and Eq. (18) at $k=1$ has a single root, $\gamma_{2}=\gamma_{2}(1,1)$, shown in the table.

Consequently, in the region of values $\gamma$

$1 \leqslant \gamma \leqslant \gamma_{2}(1,1)$

for the $\sigma$-component of linear polarization, the effective spectral width is $\Lambda=1$, where $v_{2}^{(1)}=1$. There are no other values of $\tilde{v}_{2}^{(1)}$ and $\tilde{\gamma}_{2}$ for $\Lambda=1$, which is also indicated in the table.

Next, we examine the value $\Lambda_{2}=2$. In this case, Eq. (17) has the form

$P_{2}(\beta ; k)+P_{2}(\beta ; k+1)-\frac{1}{2}=0$.

Since at the previous step it has been established that in the region $1 \leqslant \gamma \leqslant \gamma_{2}(1,1)$ the effective width is $\Lambda_{2}=1$, one is required to examine the solutions of Eq. (20) only in the region

$\gamma>\gamma_{2}(1,1)$

Analysis of Eq. (20) shows that these equations have a unique solution, $\gamma_{2}(2,1)$, only for $k=1$, where $\gamma_{2}(1,1)<\gamma_{2}(2,1)$.

Consequently, in the region of values $\gamma$

$\gamma_{2}(1,1)<\gamma \leqslant \gamma_{2}(2,1)$

the effective width is $\Lambda_{2}=2$, where $v_{2}^{(1)}=1$. There are no other values of $\tilde{v}_{2}^{(1)}$ and $\tilde{\gamma}_{2}$ for $\Lambda=2$.

At the next step, we examine the value $\Lambda_{2}=3$. Equations (17) at $\Lambda_{2}=3$ have the form

$P_{2}(\beta ; k)+P_{2}(\beta ; k+1)+P_{2}(\beta ; k+2)-\frac{1}{2}=0$,

and we should only be concerned with solutions of these equations that belong to the region

$\gamma>\gamma_{2}(2,1)$

since in the previous steps it has been found that the effective spectral width at $1 \leqslant \gamma \leqslant \gamma_{2}(2,1)$ is such that $\Lambda_{2} \leqslant 2$. It turns out that under the restriction (24) Eq. (23) possesses the solutions $\gamma_{2}(3,1)$ at $k=1$, and $\gamma_{2}(3,2)$ at $k=2$. These solutions obey the inequalities $\gamma_{2}(2,1)<\gamma_{2}(3,2)<\gamma_{2}(3,1)$.
Consequently, in the region of values $\gamma$

$\gamma_{2}(2,1)<\gamma \leqslant \gamma_{2}(3,1)$

the effective width is $\Lambda_{2}=3$, where $v_{2}^{(1)}=1$. However, inside the region (25) there is a subregion:

$\gamma_{2}(2,1)<\gamma \leqslant \gamma_{2}(3,2)<\gamma_{2}(3,1)$,

which admits a larger value, $v_{2}^{(1)}=\tilde{v}_{2}=2$, as shown in the table.

At the next step, we examine $\Lambda_{2}=4,5,6$. For these values of $\Lambda_{2}$ the results of the calculation are similar to those obtained for $\Lambda_{2}=3$, namely, these are the values of $\gamma$ that belong to the region

$\gamma_{2}\left(\Lambda_{2}-1,1\right)<\gamma \leqslant \gamma_{2}\left(\Lambda_{2}, 1\right)$

with the effective width being equal to the corresponding $\Lambda_{2}$, where $v_{2}^{(1)}=1$. However, inside the region (27) there is a subregion:

$\gamma_{2}\left(\Lambda_{2}-1,1\right)<\gamma \leqslant \gamma_{2}\left(\Lambda_{2}, 2\right)<\gamma_{2}\left(\Lambda_{2}, 1\right)$,

which admits a larger value, $v_{2}^{(1)}=\tilde{v}_{2}=2$, as shown in the table.

At the next steps, we examine $\Lambda_{2}=7 \div 11$, and each step involves solutions of Eq. (17) only in the region of values $\gamma$ that are larger than those at the previous step. For these values of $\Lambda_{2}$, Eq. (17) possesses the solutions $\gamma_{2}\left(\Lambda_{2}, v_{2}^{(1)}\right)$ corresponding to the three values $v_{2}^{(1)}=1, v_{2}^{(1)}=2, v_{2}^{(1)}=$ $\tilde{v}_{2}=3$, satisfying the inequalities

$\gamma_{2}\left(\Lambda_{2}-1, v_{2}^{(1)}\right)<\gamma_{2}\left(\Lambda_{2}, k\right)<\gamma_{2}\left(\Lambda_{2}, n\right), k>n$.

In the table, we indicate the smallest value $v_{2}^{(1)}=1$ and the respective largest value $\gamma_{2}\left(\Lambda_{2}, 1\right)$, as well as the largest value $v_{2}^{(1)}=\tilde{v}_{2}=3$ and the respective smallest value $\gamma_{2}\left(\Lambda_{2}, 3\right)$. For $\Lambda_{2}=7 \div 11$, the corresponding regions of $\gamma$ are determined by Eq. (27), but now there are two subregions: one is determined by Eq. (28), so that the values of $\gamma$ admit $v_{2}^{(1)}=2$; the other one is determined by the condition

$\gamma_{2}\left(\Lambda_{2}-1,1\right)<\gamma \leqslant \gamma_{2}\left(\Lambda_{2}, 3\right)<\gamma_{2}\left(\Lambda_{2}, 2\right)<\gamma_{2}\left(\Lambda_{2}, 1\right)$,

and this region of $\gamma$ also admits the value $v_{2}^{(1)}=3$.

The next step concerns the region $\Lambda_{2}=12 \div 27$. Here, it is essential that the smallest possible value is $v_{2}^{(1)}=2$; otherwise, the results are identical to the case $\Lambda_{2}=7 \div 11$.

In general, the column for $s=2$ of the table indicates for each $\Lambda_{2}$ the smallest possible value $v_{2}^{(1)}=v_{2}^{(1)}\left(\Lambda_{2}\right)$ and 
the corresponding largest value $\gamma_{2}=\gamma_{2}\left(\Lambda_{2}, v_{2}^{(1)}\left(\Lambda_{2}\right)\right)$, as well as the largest possible value $\tilde{\nu}_{2}$ for this $\Lambda_{2}$ and the corresponding smallest value $\tilde{\gamma}_{2}$. Possible intermediate values $v_{2}^{(1)}$ between the smallest $v_{2}^{(1)}\left(\Lambda_{2}\right)$ and the largest $\tilde{v}_{2}$, as well as the respective intermediate values $\gamma_{2}$, are not specified. The intermediate values of $\gamma_{2}$ always satisfy the relations of Eq. (29).

Consequently, in the column for $s=2$ we indicate the regions of values $\gamma$

$\gamma_{2}\left(\Lambda_{2}-1, v_{2}^{(1)}\left(\Lambda_{2}-1\right)\right)<\gamma \leqslant \gamma_{2}\left(\Lambda_{2}, v_{2}^{(1)}\left(\Lambda_{2}\right)\right)$

for which the effective spectral width for the $\sigma$-component of SR linear polarization equals $\Lambda_{2}$. We also indicate the initial points of the effective spectral width. These points are not determined uniquely. For the smallest initial value $v_{2}^{(1)}$, the range of values (31) taken by $\gamma$ is the largest one, while this region is the smallest one for the largest possible value $\tilde{v}_{2}$.

For the other polarization components, the results of the calculation are given in the respective columns of the table. In particular, the table shows that for equal values $\Lambda_{s}$ the corresponding values $\gamma_{s}$ obey the inequalities

$\gamma_{3}>\gamma_{1}>\gamma_{0}>\gamma_{2}>\gamma_{-1}$.

At a fixed energy $\gamma$, the corresponding values of $\Lambda_{s}$ are restricted by

$\Lambda_{3}<\Lambda_{1}<\Lambda_{0}<\Lambda_{2}<\Lambda_{-1}$

In this way, for each polarization component of synchrotron radiation we have found energy regions at which the effective spectral width equals $\Lambda_{s}$, and the initial harmonic of this effective width is determined. Numeric calculations have been carried out in the case $\Lambda_{s} \leqslant 100$.

In the ultra-relativistic case, the corresponding results have been obtained in [13].

Acknowledgements The work of Bagrov and Gitman is partially supported by RFBR research project No. 15-02-00293a and by Tomsk State University Competitiveness Improvement Program. Gitman thanks $\mathrm{CNPq}$ and FAPESP for their permanent support. Levin thanks CNPq for permanent support.

Open Access This article is distributed under the terms of the Creative Commons Attribution 4.0 International License (http://creativecomm ons.org/licenses/by/4.0/), which permits unrestricted use, distribution, and reproduction in any medium, provided you give appropriate credit to the original author(s) and the source, provide a link to the Creative Commons license, and indicate if changes were made.

Funded by SCOAP ${ }^{3}$.

\section{References}

1. A.A. Sokolov, I.M. Ternov, Synchrotron Radiation (Akademie, Berlin, 1968)

2. H. Winick, S. Doniach, Synchrotron Radiation Research (Plenum Press, New York, 1980)

3. A.A. Sokolov, I.M. Ternov, C.W. Kilmister, Radiation from Relativistic Electrons (American Institute of Physics, New York, 1986)

4. V.G. Bagrov et al., Synchrotron Radiation Theory and Its Development (World Scientific, Singapore, 1999)

5. P.J. Duke, Synchrotron Radiation: Production and Properties (Oxford University Press, Oxford, 2000)

6. H. Wiedemann, Synchrotron Radiation (Springer, Berlin, 2003)

7. A. Hofmann, The Physics of Synchrotron Radiation (Cambridge University Press, Cambridge, 2004)

8. V.G. Bagrov, M.V. Dolzhin, V.B. Tlyachev, A.T. Jarovoi, Evolution of the angular distribution of circularly polarized synchrotron radiation with charge energy. Russ. Phys. J. 47(4), 414-423 (2004). doi:10.1023/B:RUPJ.0000042770.97166.87

9. V.G. Bagrov, D.M. Gitman, V.B. Tlyachev, A.T. Jarovoi, Evolution of angular distribution of polarization components for synchrotron radiation under changes of particle energy, in International Summer School: Recent Problems in Field Theory, vol. 4, ed. by A.V. Aminovain, K. Heter (Kazan, Petrov School, 2004), pp. 9-24. (in Russian)

10. V.G. Bagrov, D.M. Gitman, V.B. Tlyachev, A.T. Jarovoi, Evolution of angular distribution of polarization components for synchrotron radiation under changes of particle energy, in International Conference: Particle Physics in Laboratory, Space and Universe. Moscow State University, Faculty Physics, ed. by A.I. Studenikin (World Scientific, New Jersey, 2005), pp. 355-362

11. V.G. Bagrov, D.M. Gitman, V.B. Tlyachev, A.T. Jarovoi, New theoretical results in synchrotron radiation. Nucl. Instrum. Methods Phys. Res. B 240(3), 638-645 (2005). doi:10.1016/j.nimb.2005. 03.286

12. V.G. Bagrov, M.V. Dolzhin, K.G. Seravkin, V.M. Shakhmatov, Partial contributions of individual harmonics to the power of synchrotron radiation. Russ. Phys. J. 49(7), 681-689 (2006). doi:10. 1007/s11182-006-0162-1

13. V.G. Bagrov, D.M. Gitman, A.D. Levin, A.S. Loginov, A.D. Saprykin, Effective spectrum width of the synchrotron radiation. Eur. Phys. J. C 75(11), 555 (2015). doi:10.1140/epjc/ s10052-015-3798-6

14. V. G. Bagrov, D. M. Gitman, A. D. Levin, A. S. Loginov, A. D. Saprykin, Dependence of Effective spectrum width of synchrotron radiation on particle energy. arXiv:1703.02695v1 [physics.class$\mathrm{ph}]$ (2017) 